\title{
Damage-controlled high power lasers and plasma mirror application
}

\section{Hiromitsu Kiriyama, Yoshihiro Ochi, Masaharu Nishikino, Keisuke Nagashima, Tetsuya Kawachi, et al.}

Hiromitsu Kiriyama, Yoshihiro Ochi, Masaharu Nishikino, Keisuke Nagashima, Tetsuya Kawachi, Ryoji Itakura, Akira Sugiyama, Masaki Kando, A. S. Pirozhkov, Mamiko Nishiuchi, Sergei V. Bulanov, Kimonori Kondo, Yoshiaki Kato, "Damage-controlled high power lasers and plasma mirror application," Proc. SPIE 9532, Pacific Rim Laser Damage 2015: Optical Materials for HighPower Lasers, 95320A (5 August 2015); doi: 10.1117/12.2185763 


\title{
Damage-controlled high power lasers and plasma mirror application
}

Hiromitsu Kiriyama ${ }^{a}$, Yoshihiro Ochi ${ }^{a}$, Masaharu Nishikino ${ }^{a}$, Keisuke Nagashima ${ }^{a}$, Tetsuya Kawachi $^{\mathrm{a}}$, Ryoji Itakura ${ }^{\mathrm{a}}$, Akira Sugiyama ${ }^{\mathrm{a}}$, Masaki Kando ${ }^{\mathrm{a}}$, A.S.Pirozhkova ${ }^{\mathrm{a}}$, Mamiko Nishiuchi ${ }^{\mathrm{a}}$, Sergei V. Bulanov ${ }^{\mathrm{a}}$, Kiminori Kondo ${ }^{\mathrm{a}}$, Yoshiaki Kato*b, a

${ }^{a}$ Quantum Beam Science Center, Kansai Photon Science Institute, Japan Atomic Energy Agency, Kizugawa, Kyoto, Japan 619-0215; ' $T$ The Graduate School for the Creation of New Photonics Industries, Hamamatsu, Shizuoka, Japan 431-1202

\begin{abstract}
Following three different types of high power lasers at Kansai Photon Science Institute are overviewed and controlling the laser damages in these laser systems are described: (1) PW-class Ti:sapphire laser for high field science, (2) zig-zag slab Nd:glass laser for x-ray laser pumping, and (3) high-repetition Yb:YAG thin-slab laser for THz generation. Also reported is the use of plasma mirror for characterization of short-wavelength ultrashort laser pulses. This new method will be useful to study evolution of plasma formation which leads to laser damages.
\end{abstract}

Keywords: laser damage, Ti:sapphire laser, zig-zag slab amplifier, thin-slab laser, x-ray laser, THz generation, plasma mirror, PM-FROG

\section{INTRODUCTION}

At Kansai Photon Science Institute (KPSI), Japan Atomic Energy Agency (JAEA), various types of high power lasers have been developed to generate high power x-ray, gamma-ray, $\mathrm{THz}$ radiation and particle beams, for applications to science, medicine and industry. Controlling the laser damage in high power laser systems is one of the most important issues in realizing various applications. In this paper, we report on different types of high power lasers and their applications at KPSI and describe how the laser damage is controlled in these laser systems. Also reported is the use of the plasma mirror for characterization of short-wavelength ultrashort laser pulses. This new method, called PM-FROG, will be useful for studying the evolution of laser damages.

\section{ULTRASHORT PULSE LASER FOR HIGH FIELD SCIENCE: J-KAREN}

When the laser intensity becomes comparable to or exceeds the relativistic intensity, where the velocity of electrons driven the laser field becomes close to the speed of light, various new nonlinear phenomena take place, such as relativistic self-focusing of the laser beam, electron acceleration to high energies by the laser-driven plasma wake field, and high energy ion generation due to intense field generated by the electrons. This relativistic intensity is approximately $2 \times 10^{18} \mathrm{~W} / \mathrm{cm}^{2}$ at the laser wavelength of $800 \mathrm{~nm}$. At higher laser intensities exceeding $\sim 10^{22} \mathrm{~W} / \mathrm{cm}^{2}$, protons will be efficiently accelerated to high energies due to ponderomotive force to the protons, and nonlinear effects in vacuum will start to take place at even higher intensities [1].

At KPSI, extensive research has been undertaken on high field science using the ultrashort-pulse high intensity Ti:sapphire lasers. Recent results include laser frequency upshifting from $800 \mathrm{~nm}$ to $37 \mathrm{~nm}$ due to reflection from a relativistic plasma mirror [2], high-order harmonics generation reaching to the water window region from relativistic electron spikes [3], and acceleration of highly charged Fe ions to $\mathrm{GeV}$ energies [4]. This generation of high energy, highly charged heavy ions with the intense laser field may lead to a new approach for laser-driven extraction and acceleration of exotic nuclei, especially of unstable nuclei with extremely short life times, which cannot be investigated with present high energy ion accelerators [4].

J-KAREN is a PW-class Ti:sapphire laser developed at KPSI [5]. This laser system, after initial operation in 2000, has been improved to reduce prepulses for higher contrast to $\sim 2 \times 10^{12}$ by introducing optical parametric chirped-pulse

*y.kato@gpi.ac.jp; phone +81-53-484-2501; fax +81-53-487-3012

Pacific Rim Laser Damage 2015: Optical Materials for High-Power Lasers, edited by Jianda Shao,

Takahisa Jitsuno, Wolfgang Rudolph, Proc. of SPIE Vol. 9532, 95320A · C 2015 SPIE

CCC code: $0277-786 X / 15 / \$ 18 \cdot$ doi: $10.1117 / 12.2185763$ 


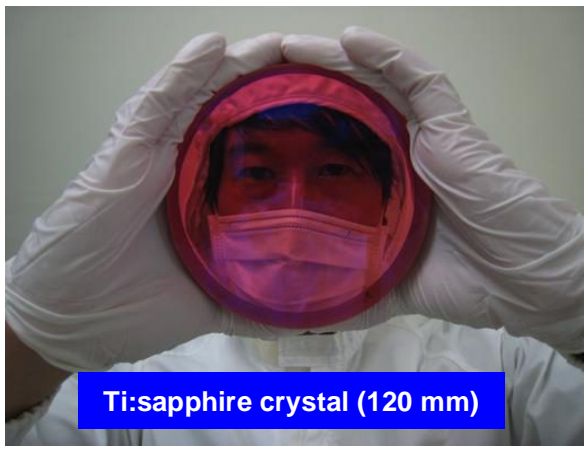

(a)

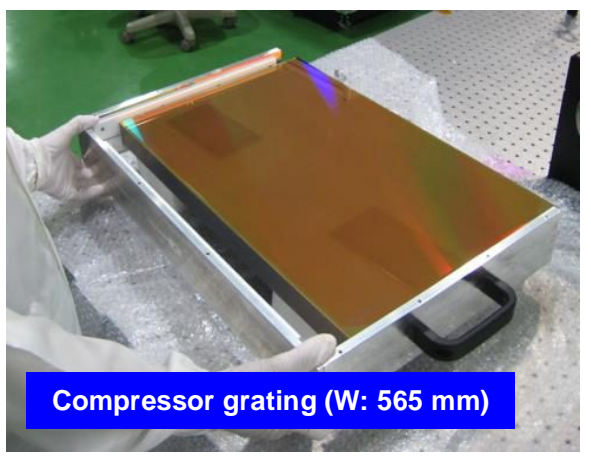

(b)

Figure 1. Photographs of (a) a 120-mm diameter Ti:sapphire crystal and (b) a gold-coated diffraction grating with 565-mm width used for upgrading J-KAREN laser.

amplifier (OPCPA) in the front-end and to generate a flat-top spatial profile in the final amplifier with a 80-mm diameter Ti:sapphire crystal by improving the pump beam spatial profile.

Upgrading of J-KAREN to higher power, J-KAREN-P, is undertaken by adding a 120-mm diameter Ti:sapphire crystal as a booster amplifier. This booster amplifier is pumped by 6 flash-lamp pumped Nd:glass lasers, each with $25 \mathrm{~J}$ energy at 532-nm with a repetition rate of $0.1 \mathrm{~Hz}$. The amplified beam of 80-mm diameter is expanded to 200-mm diameter in order to reduce the fluence at the pulse compressor. Large aperture gold coated diffraction gratings with $565 \mathrm{~mm}$ width are used for compression of the high energy chirped pulses of over 55-J energy. After pulse compression, this system is expected to generate a high-contrast laser pulse with over 30-J energy and $30 \mathrm{fs}$ pulse duration, exceeding 1-PW peak power, with a repetition rate of $0.1 \mathrm{~Hz}$. Fig. 1 shows a $120-\mathrm{mm}$ Ti:sapphire crystal and a 564-mm width diffraction grating used for J-KAREN-P.

The weakest part in this laser system in terms of laser damage is the gold-coated diffraction grating whose damage threshold is approximately $0.2 \mathrm{~J} / \mathrm{cm}^{2}$. The ultrashort, high power laser systems will become more compact, if dispersive elements with higher damage threshold are developed. Although multilayer diffraction gratings have higher damage threshold, its bandwidth is not broad enough at present for the Ti:sapphire laser systems. Another possibility is to improve the bandwidth and damage threshold of the transmission gratings, where high diffraction efficiency of almost $98 \%$ has been demonstrated.

\section{ZIG-ZAG SLAB AMPLIFIERES FOR X-RAY LASER PUMPING: TOPAZ}

In spite of rapid development of various types of solid state lasers, the Nd:glass lasers are still important for high energy lasers systems, because Nd-doped glasses have high stored energy densities and large aperture Nd:glasses can be easily fabricated. We have developed a zig-zag slab Nd:glass laser system for pumping plasma X-ray lasers [6]. In order to pump the oscillator and the amplifier of the $\mathrm{x}$-ray laser system, two lasers beams each with $\sim 10 \mathrm{~J}$ laser energy are required. Since this $\mathrm{x}$-ray laser is used for various applications, high-repetition operation is desirable to supply the $\mathrm{x}$-ray laser beam to many users.

For repetitive-operation of the laser with the Nd:glass, which has low thermal conductivity, we have developed the slabgeometry amplifiers where the laser slabs are cooled by water at their broad surfaces for efficient cooling. The slabs are pumped by water-cooled flash lamps through the broad surface, and the laser beam is propagated in the slab along a zigzag path by total reflection at the glass-water boundaries. Two types of slab amplifiers have been developed; a smaller amplifier (ZSA-1) with 17-mm x 99-mm x 15-mm-thick Nd:glass for a pre-amplifier and a larger amplifier (ZSA-2) with 110-mm x 205-mm x 15-mm-thick Nd:glass for a main amplifier. 


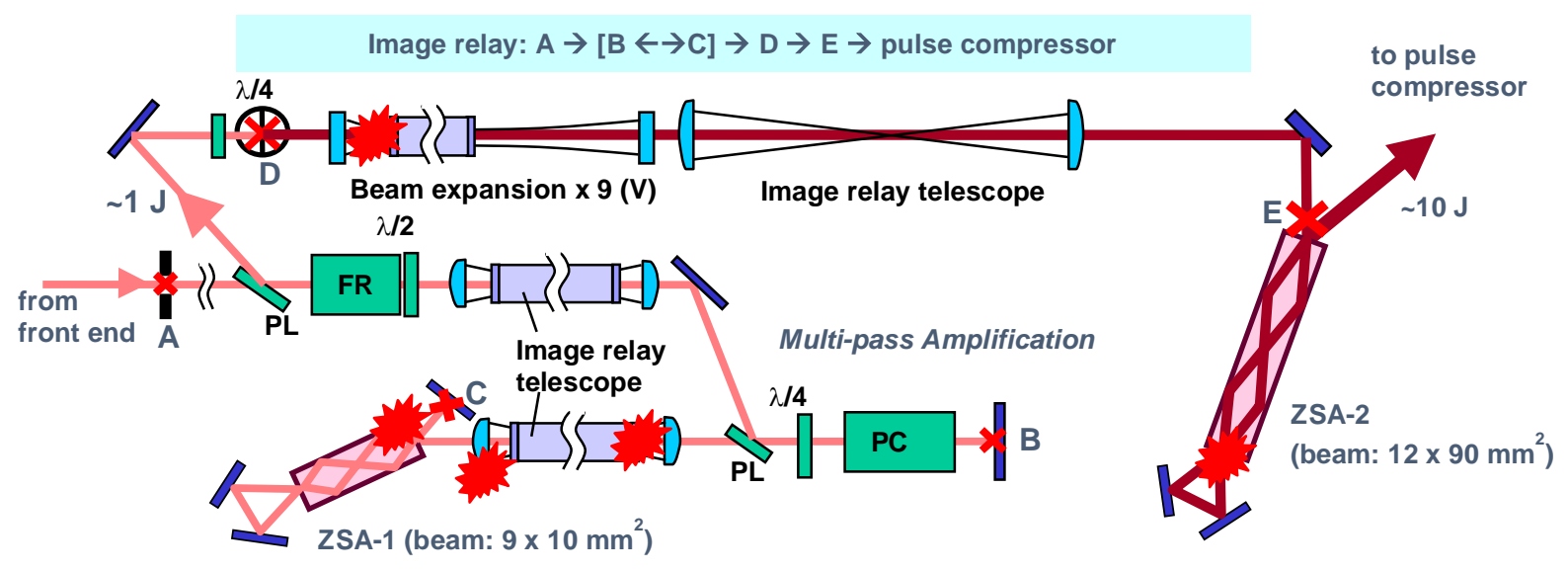

Figure 2. Optical layout of one beam of the zig-zag slab laser system TOPAZ for x-ray laser pumping. The rectangular beam pattern at the soft aperture at $\mathrm{A}$ is relayed to B, C, D and E by the image relay telescopes. The locations susceptible to laser damage are marked by stars. Acronyms are PL: polarizer, $\lambda / 2$ : half-wave waveplate, $\lambda / 4$ : quarter-wave waveplate, FR: Faraday rotator, and PC: Pockels cell.

Layout of a part of the 2-beam laser system is shown in Fig. 2. The laser beam from the front-end is injected into the preamplifier line of "cavity-dump" geometry, where the laser beam is amplified 4 times in ZSA-1. Then it is amplified twice in ZSA-2. The circular beam from the front end is shaped into a rectangular pattern with a rectangular soft aperture (SA), and image-relayed and expanded with the spherical lens pairs (SLP) and the cylindrical lens pairs (CLP). Particular caution was taken to obtain uniform beam patterns to achieve large filling factor and efficient energy extraction at the amplifiers, and yet not to irradiate the edges of the amplifiers in order to avoid the laser damage to the amplifiers and also to reduce diffraction which leads to non-uniform beam patterns in the downstream. The critical places in this laser system in terms of the laser damage are also marked in Fig. 2. The laser beam after the final amplifier with 14-J energy and 1-ns pulse width is sent to a pulse compressor composed of 2 diffraction gratings. After pulse compression, the laser beam has 10-J/beam energy and 500-fs pulse width.

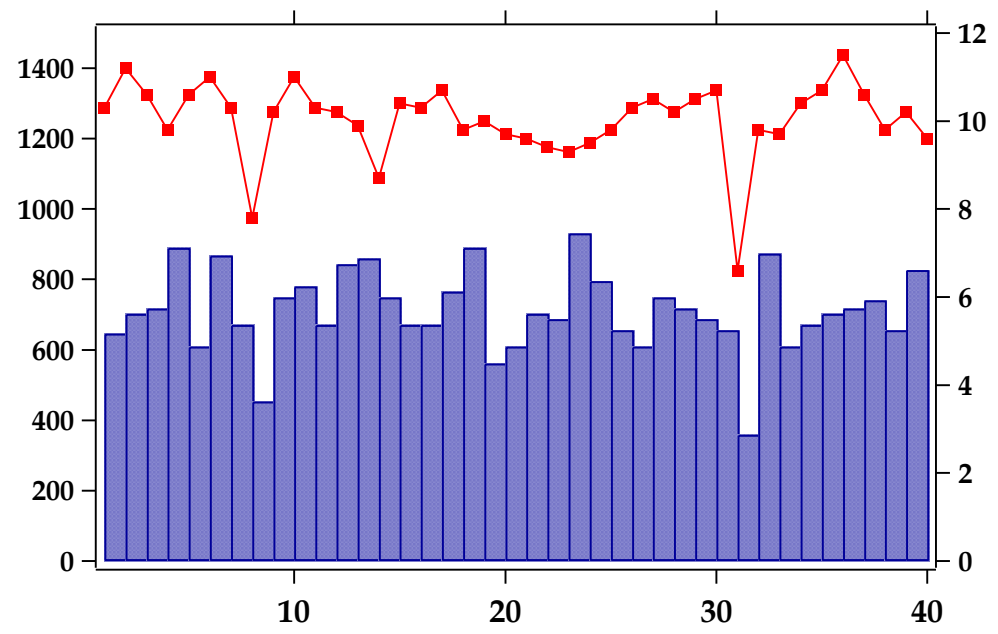

Figure 3. Reproducibility of the pump laser energy for the second target (vertical bars) and the x-ray laser energy (red closed circles). The vertical scale on the right-hand side shows the pump laser energy in J, and the vertical scale on the left-hand side shows the $\mathrm{x}$-ray laser energy in $\mathrm{nJ}$ normalized by the divergence in $\mathrm{mrad}^{2}$. The horizontal axis is the consecutive shot number. 
The x-ray laser target is a thin Ag plate, which is irradiated with the Nd:glass laser beam [7]. The pulse front of the pumping laser is tilted for travelling wave amplification of the soft $\mathrm{x}$-ray laser of $13.9 \mathrm{~nm}$ wavelength in the Ag plasma. The x-ray laser is composed of an oscillator and an amplifier, where a part of the x-ray laser beam from the oscillator is amplified in the amplifier to generate a very small divergence beam with almost full spatial coherence [8]. Typical energy and the divergence of the amplified x-ray laser is $600 \mathrm{~nJ}$ and $0.3 \mathrm{mrad}$, respectively. Figure 3 shows the reproducibility of the energies of the pump laser and the x-ray laser, when this system is repetitively operated at $0.1 \mathrm{~Hz}$. Although there still remain fluctuations, this $x$-ray laser system is very useful for quantitative experiments in various applications.

\section{1-KHZ, THIN DISK LASER FOR THZ GENRERATION: QUADRA-T}

High repetition solid state lasers are useful for broad range of applications in science, industry and medicine. We have developed a 1-kHz, 10-W, 1- $\mu \mathrm{m}$ laser for generating high power THz radiation. The Yb:YAG thin-disk ceramic used as an amplifier has $200-\mu \mathrm{m}$ thickness and 10-mm outer diameter. This disk is doped with 7 atomic $\% \mathrm{Yb}$ in the central area of 6-mm diameter. The optical layout of this amplifier is shown in Fig. 4. The thin disk is pumped with cw laser diodes of 940-nm wavelength. A seed pulse of 1030-nm wavelength and 500-ps duration from the front end is double passed in the thin disk amplifier. The thin disk is anti-reflection (AR) coated on the front surface and high reflection (HR) coated at the back surface which is in close contact with a Cu heat sink. High damage thresholds are required to both of AR and HR coatings for reliable operation of the laser system.

The flux of the amplified pulse is estimated to be approximately $2 \mathrm{~J} / \mathrm{cm}^{2}$. This flux increases 4 times at the front surface of the thin disk due to overlap of the laser pulse in the amplifier. We have targeted the laser damage threshold of the AR coating at $40 \mathrm{~J} / \mathrm{cm}^{2}$ for a 500 -ps laser pulse, including the safety factor of 5 considering the laser beam non-uniformity and other effects. Similar damage threshold is required also to the HR coating at the back surface of the thin disk. The $\mathrm{Al}_{2} \mathrm{O}_{3} / \mathrm{SiO}_{2}$ multilayers were used for the AR- and HR-coatings [9]. Non-quarter wave layer was coated on the multilayer in order to shift the peak optical field from the $\mathrm{Al}_{2} \mathrm{O}_{3}$ layer to the $\mathrm{SiO}_{2}$ layer in order to increase the damage threshold. The measured damage threshold is approximately $75 \mathrm{~J} / \mathrm{cm}^{2}$ for the AR coating and $124 \mathrm{~J} / \mathrm{cm}^{2}$ for the HR coating, both at $500 \mathrm{ps}$ pulse duration.

The 500-ps laser pulse is compressed to 1.3 ps and sent to a nonlinear crystal to generate $\mathrm{THz}$ radiation by optical rectification, where $\mathrm{THz}$ radiation is generated as difference frequencies from a broad band-width laser pulse. The pulse front was tilted to phase match the pumping pulse with the $\mathrm{THz}$ beam in a $\mathrm{LiNbO}_{3}$ crystal [10]. The temporal pulse shape, the spectrum and the output power of the $\mathrm{THz}$ radiation thus generated are shown in Fig. 5. With the pump power of $7 \mathrm{~W}$, THz radiation of $4 \mathrm{~mW}$ has been generated with high stability. The THz spectrum is peaked at $0.3 \mathrm{THz}$ and covers $0.1 \sim 0.8 \mathrm{THz}$. This spectrum could be extended by broadening the bandwidth of the pumping laser which is 1.24 $\mathrm{nm}$ at present, narrowed from $4 \mathrm{~nm}$ of the input pulse during amplification in the $\mathrm{Yb}$ :YAG amplifier. It is planned to upgrade this thin disk laser to $100 \mathrm{~W}$ by adding an amplifier with a larger aperture.

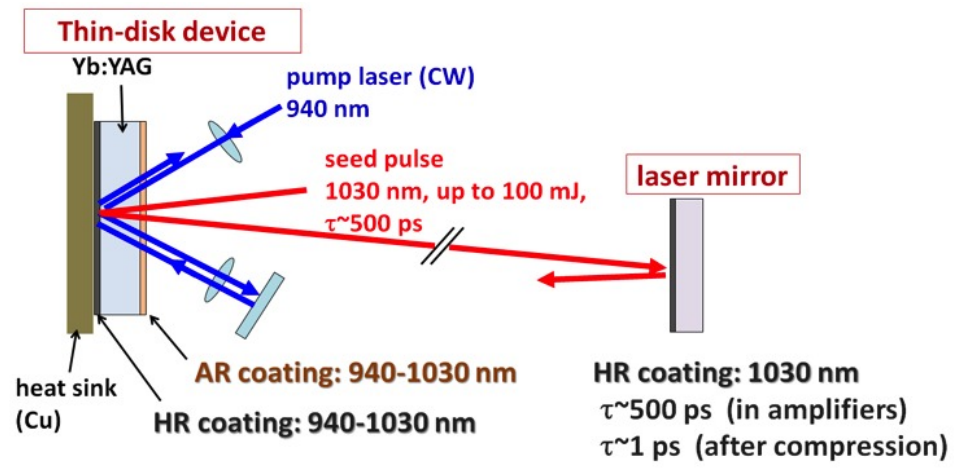

Figure 4. Layout of a Yb:YAG ceramic thin-disk amplifier pumped by $\mathrm{cw}$ LD at $940 \mathrm{~nm}$. The thin disk has 200- $\mu \mathrm{m}$ thickness and 10-mm diameter, in which central area of 6-mm diameter is doped with $\mathrm{Yb}$. 

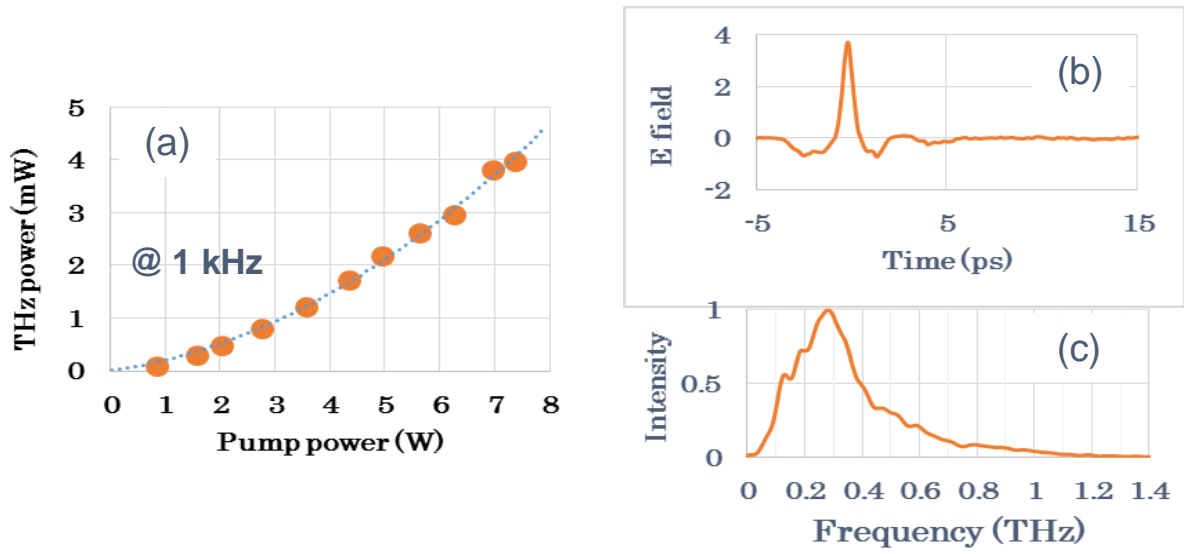

Figure 5. Properties of $\mathrm{THz}$ radiation generated with QUADRA-T laser: (a) THz power vs pump laser power, (b) time dependence of the electric field of THz radiation, and (c) spectral intensity of THz radiation. (Vertical axes in (b) and (c) are in arbitrary scales.)

\section{PLASMA MIRROR FROG}

Plasma mirror is an optical device, where a transparent $\mathrm{SiO}_{2}$ plate is used to reduce prepulses from a high intensity laser pulse by plasma formation on the $\mathrm{SiO}_{2}$ surface. When the density of the plasma produced by the prepulses exceeds the critical density, the plasma becomes reflective in a short time and reflects only the main laser pulse.

We have developed a new method named PM-FROG, plasma mirror FROG (frequency-resolved optical gating), to measure the time-dependent intensity and phase of ultrashort-duration vuv pulses [11]. Plasma mirror is useful in this application since the plasma is reflective even to vuv radiation, whereas transparent optical materials required for vuv pulse characterization with other methods are not easily available in this short wavelength region. We have applied this new method to characterize an ultrashort pulse of 160-nm wavelength, which is generated as the fifth harmonic of a 795$\mathrm{nm}, 60$-fs pulse from a Ti:sapphire laser.

In PM-FROG, a $\mathrm{SiO}_{2}$ surface is irradiated with a high-intensity ultrashort pulse (pump pulse) to produce a plasma. After time delay $\tau$, a vuv pulse to be measured (probe pulse) is reflected by the plasma mirror and the spectrum of the reflected pulse is measured with a vuv spectrometer. By changing $\tau$, the time-dependent spectrum of the probe pulse is measured as shown schematically in Fig. 6.

This measured spectrum is given by the Fourier transform of the convolution of the amplitude of the probe pulse, $E(t)$, and the reflectivity of the plasma mirror, $R(t-\tau)$, as

$$
I_{F R O G}(\omega, \tau)=\left|E_{\text {sig }}(\omega, \tau)\right|^{2}=\left|\int E(t) \sqrt{R(t-\tau)} \exp (-i \omega t) d t\right|^{2} .
$$

The plasma reflectivity $R(t)$ is constant for $t \geqq 0$ and 0 for $t<0$ in an ideal case. The time dependences of the complex field of the probe pulse and also the reflectivity of the plasma mirror can be determined by iterative calculation using PCGPA (principal component generalized projection algorithm) until good agreement is obtained between the measured spectrum and the retrieved spectrum, as shown in Fig. 6. The time- and the spectral-dependences of the intensity and the phase of the probe pulse, as well as the time dependence of the reflectivity of the plasma mirror, determined from the iterative calculation, are shown in Fig. 7. The spectral phase change is mostly attributed to the dispersion of the LiF lens used in the vuv beam. Here we find that the reflectivity of plasma mirror has a rise time of approximately 60 fs and takes almost a constant value overlapped with slight temporal modulation.

This work has shown that PM-FROG is useful to characterize the complex waveforms of ultrashort pulses in vuv wavelength regions. The time dependence of the reflectivity of the plasma mirror has been also determined with this 

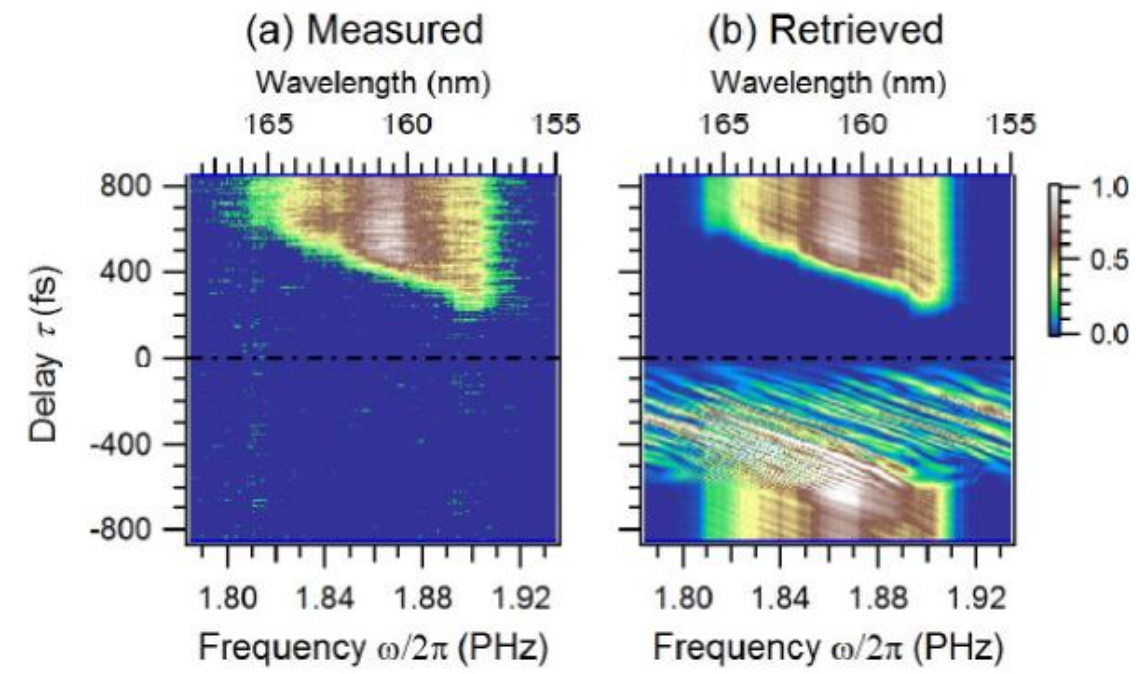

Figure 6. Time-resolved reflection spectra of the vuv pulse. (a) shows the measured PM-FROG trace, whereas (b) shows the retrieved FROG trace after iterative calculation with PCGPA. The noise at $\tau<0$ in (b) is an artifact since only $\tau \geqq 0$ has been retrieved in this calculation.
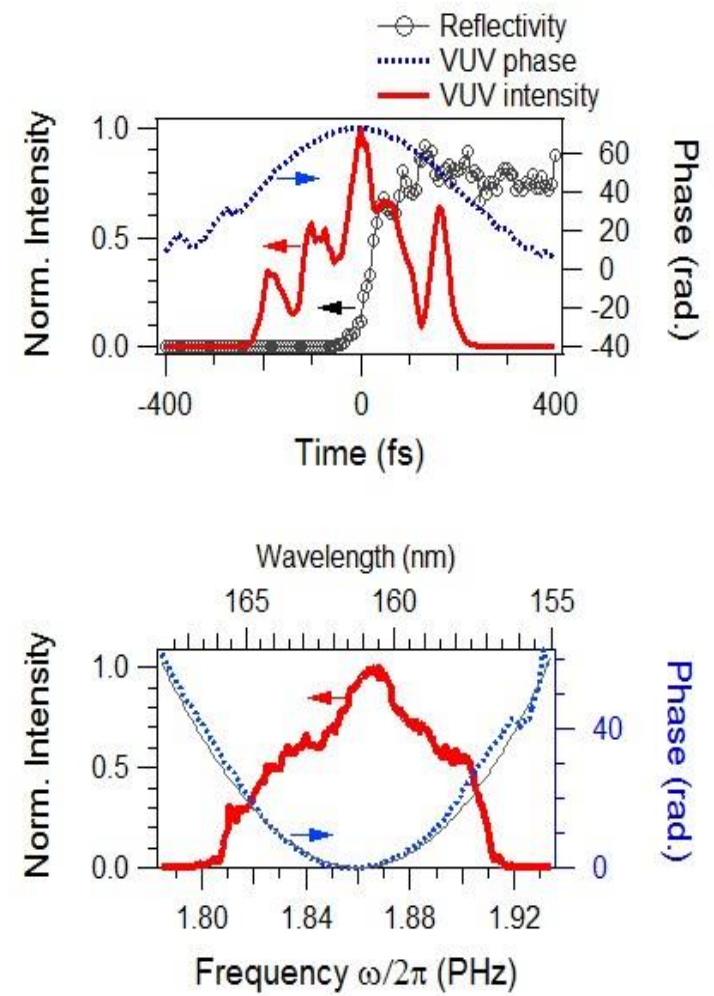

Figure 7. Upper figure shows the time-resolved intensity (solid curve) and phase (dotted curve) of the vuv pulse and the time-resolved reflectivity of the plasma mirror (open circles), both determined by PM-FROG. Lower figure shows the frequency dependence of the intensity (solid curve) and phase (dotted curve) of the vuv pulse, where the thin solid curve shows the calculated phase change of the vuv pulse due to propagation in a LiF lens of $2.3 \mathrm{~mm}$ thickness. 
method. In order to extend this method to other wavelengths, especially to shorter wavelengths, it is necessary in future to investigate the wavelength dependence of the reflectivity of the plasma mirror. This in turn will lead to the study of plasma formation process on the $\mathrm{SiO}_{2}$ surface with the pump pulse.

\section{SUMMARY}

High power laser systems developed at Kansai Photon Science Institute have been overviewed, and several issues on laser damage in these laser systems have been presented. These laser systems are (1) a PW-class high peak power ultrashort-pulse Ti:sapphire laser for high field science, (2) a 2 beam, 10-J/beam, $0.1 \mathrm{~Hz}$ laser system using zig-zag slab $\mathrm{Nd}$ :glass amplifiers for $\mathrm{x}$-ray laser pumping, and (3) a 1-kHz, 10-W, 1- $\mu$ m laser system using thin Yb:YAG ceramic disk amplifiers for $\mathrm{THz}$ generation.

Considering the importance of laser damage control for reliable and high performance operation of the laser systems, some general observation can be made. Since the damage to optical components occurs at the locations of high fluence, laser beam profile has to be controlled over the whole laser system to minimize spatial non-uniformities using, for example, spatial filtering, image relaying and soft apertures to avoid diffraction at the edges of optical components. Reduction of diffraction due to local non-uniformities such as damage of optical components and dust particles are also important in real laser systems.

The laser damage threshold of optical components depend critically on many factors; laser wavelength, pulse duration, peak power for pulsed lasers, average power for $\mathrm{cw}$ and high repetition pulsed lasers, anti-reflection surfaces, high reflection surfaces, and so on. The optical components have to be used well below their damage thresholds for reliable long-term operation of the laser system. Also the performance of the laser system, such as laser power, temporal pulse shapes and beam profiles, has to be continuously monitored to detect any changes in the laser system, which often lead to serious degradation of the laser system.

In this paper, we have also introduced a new method PM-FROG, with which ultrashort pulses at vuv wavelength region can be characterized by using a plasma mirror. With PM-FROG, not only the intensity and phase of the short wavelength pulses are measured, but also the temporal change of the reflectivity of the plasma mirror can be determined. This method will be useful to investigate detailed processes of plasma formation and subsequent evolution to laser damages in various conditions.

\section{REFERENCES}

[1] Mourou, G. A., Tajima, T., and Bulanov, S. V., "Optics in the relativistic regime", Rev. Mod. Phys. 78, 309 (2006).

[2] Kando, M., Pirozhkov, A., Kawase, K., Esirkepov, T., Fukuda, Y., Kiriyama, H., Okada, H., Daito, I., Kameshima, T., Hayashi, Y., Kotaki, H., Mori, M., Koga, J., Daido, H., Faenov, A., Pikuz, T., Ma, J., Chen, L., Ragozin, E., Kawachi, T., Kato,T., Tajima, T., and Bulanov, S., "Enhancement of photon number reflected by the relativistic flying mirror", Phys. Rev. Lett. 103, 235003 (2009).

[3] Pirozhkov, A. S., Kando, M., Esirkepov, T. Zh., Gallegos, P., Ahmed, H., Ragozin, E. N., Faenov, A. Ya., Pikuz, T. A., Kawachi, T., Sagisaka, A., Koga, J. K., Coury, M., Green, J., Foster, P., Brenner, C. , Dromey, B., Symes, D. R., Mori, M., Kawase, K., Kameshima, T., Fukuda, Y., Chen, L., Daito, I., Ogura, K., Hayashi, Y., Kotaki, H., Kiriyama, H., Okada, H., Nishimori, N., Imazono, T., Kondo, K., Kimura, T., Tajima, T., Daido, H., Rajeev, P., McKenna, P., Borghesi, M., Neely, D., Kato, Y., and Bulanov, S. V., "Soft-X-ray harmonic comb from relativistic electron spikes", Phys. Rev. Lett. 108, 135004 (2012).

[4] Nishiuchi, M., Sasaki, H., Esirkepov, T. Zh., Nishio, K., Pikuz, T. A., Faenov, A. Ya., Skobelev, I. Yu., Orlandi, R., Sako, H., Pirozhkov, A. S., Matsukawa, K., Sagisaka, A., Ogura, K., Kanasaki, M., Kiriyama, H., Fukuda, Y., Koura, H., Kando, M., Yamauchi, T., Watanabe, Y., Bulanov, S. V., Kondo, K., Imai, K., and Nagamiya, S., "Acceleration of highly charged GeV Fe ions from a low-Z substrate by intense femtosecond laser", Phys. Plasmas, 22, 033107 (2015).

[5] Kiriyama, H., Mori, M., Pirozhkov, A. S., Ogura, K., Sagisaka, A., Kon, A., Esirkepov, T. Zh., Hayashi, Y., Kotaki, H., Kanasaki, M., Sasaki, H., Fukuda, Y., Koga, J., Nishiuchi, M., Kando, M., Bulanov, S. V., Kondo, K., Bolton, P. 
L,. Slezak, O., Vojna, D., Sawicka-Chyla, M., Jambunathan, V., Lucianetti, A., and Mocek, T., "High-contrast, high-intensity petawatt-class laser and applications”, IEEE J. Selected Topics in Quant. Electr., 21, 160118 (2015).

[6] Ochi, Y., Hasegawa, N., Kawachi, T., and Nagashima, K., "Development of a chirped pulse amplification laser with zig-zag slab Nd:glass amlifiers dedicated to X-ray laser research", Appl. Opt. 46, 1500 (2007).

[7] Ochi, Y., Kawachi, T., Hasegawa, N., Nishikino, M., Ohba, T., Tanaka, M., Kishimoto, M., Kaihori, T., Nagashima, K., and Sugiyama, A., "Demonstration of submicro-joule, spatially coherent soft x-ray laser pumped by $0.1 \mathrm{~Hz}, 10$ joule, picosecond laser", Jpn. J. Appl. Phys. 48, 120212 (2009).

[8] Nishikino, M., Ochi, Y., Hasegawa, N., Kawachi, T., H. Yamatani, T., Ohba, T., T. Kaihori, T., and Nagashima, K., "Demonstration of a highly coherent $13.9 \mathrm{~nm}$ x-ray laser from a silver tape target", Rev. Sci. Instrum., 80, 116102 (2009).

[9] Ochi, Y., Nagashima, K., Okada, H., Tanaka, M., Tateno, R., Furukawa, R., and Sugiyama, A., "Development of high resistance anti-reflection coating by using $\mathrm{Al}_{2} \mathrm{O}_{3} / \mathrm{SiO}_{2}$ multilayer", Proc. SPIE, 8885, $88851 \mathrm{Z}$ (2013).

[10] Nagashima, K. and Kosuge, A., "Design of rectangular transmission gratings fabricated in LiNbO3 for high-power terahertz-wave generation", Jpn. J. Appl. Phys. 49, 122504 (2010); ibd. 51, 119201 (2012) (erratum).

[11] Itakura, R., Kumada, T., Nakano, M., and Akagi, H., "Frequency-resolved optical gating for characterization of vuv pulses using ultrafast plasma mirror switching”, Opt. Exp. 23, 10914 (2015). 